\title{
Um anjo para ELA: arquitetura baseada em visão computacional aplicada ao monitoramento on-line de parâmetros respiratórios de pacientes com Esclerose Lateral Amiotrófica (ELA) em ambiente hospitalar
}

\author{
Gustavo Henrique Souto da Silva, Antônio Higor Freire de Morais*, Ricardo Alexsandro de \\ Medeiros Valentim, Rummenigge Rudson Dantas, Leila Raulino Câmara Cavalcanti, Mário Emílio \\ Teixeira Dourado Júnior
}

Resumo Introdução: A Esclerose Lateral Amiotrófica (ELA) é uma doença neurodegenerativa, caracterizada por uma progressiva e fatal perda de neurônios motores do córtex cerebral, tronco encefálico e medula espinhal, mas que mantém preservada a atividade intelectual e cognitiva do paciente. Pacientes acometidos por essa doença irão invariavelmente necessitar do auxílio de ventiladores mecânicos. Métodos: Foi utilizado um conjunto de hardware e software para realizar o monitoramento dos parâmetros respiratórios dos pacientes em leitos hospitalares como forma de auxiliar à equipe de saúde. O monitoramento desses parâmetros deu-se por meio de uma webcam, que capturava os valores exibidos na tela do ventilador mecânico, e do emprego de técnicas de visão computacional e Optical Character Recognition (OCR). Neste sentido, o sistema foi testado sob três condições de luminosidade diferentes para verificar a eficácia do mesmo. Resultados: $\mathrm{O}$ sistema apresentou uma média geral de acertos de $94.90 \%$. Além disso, quando a interferência luminosa foi mínima, o sistema obteve uma média geral de acertos de $97,76 \%$. Conclusão: A adoção de um sistema computacional baseado em visão computacional para auxílio da equipe de saúde no monitoramento hospitalar de pacientes com ELA mostrou-se satisfatória. No entanto, a pesquisa mostrou que a adoção de um sistema com maior imunidade à interferências luminosas externas tende a apresentar melhores resultados.

Palavras-chave Monitoramento de pacientes, Esclerose Lateral Amiotrófica, BiPAP, Visão computacional, Ambiente WEB, Computador de placa única.

\section{An angel for ALS: architecture based on computer vision applied for on-line monitoring respiratory parameters of patients with Amyotrophic Lateral Sclerosis (ALS) in hospital environment}

\begin{abstract}
Introduction: Amyotrophic Lateral Sclerosis (ALS) is a neurodegenerative disease characterized by a progressive and fatal loss of motor neurons in the cerebral cortex, brainstem and spinal cord. In spite of that, the patient's intellectual and cognitive activity remains preserved. Patients affected by this disease will invariably need the help of mechanical ventilators. Methods: A set of hardware and software was used to perform the monitoring of respiratory parameters of patients in hospital beds as a means of assisting the healthcare team. The monitoring of these parameters was performed by a webcam that captured the values displayed on the screen of the ventilator, and the employment of computer vision techniques and Optical Character Recognition (OCR). In this sense, the system was tested under three different lighting conditions to verify its effectiveness. Results: The system presented an overall average of $94.90 \%$ of correct answers. Furthermore, when the luminous interference was minimum, it achieved an overall average of success of $97.76 \%$. Conclusion: The adoption of a computational system based on computer vision to aid the healthcare team in hospital monitoring of patients with ALS was satisfactory. However, the research has shown that the adoption of a system with greater immunity to external light interference tends to achieve better results.
\end{abstract}

Keyworks Patient monitoring, Amyotrophic Lateral Sclerosis, BiPAP, Computer vision, WEB Environment, Single-board computer.

*e-mail: higormorais@gmail.com 


\section{Introdução}

Segundo Valentim et al. (2012) a automação hospitalar é uma área multidisciplinar que envolve linguagens de programação (software), plataformas eletrônicas (hardware), atuação (mecânica) e fluidos fármacos. A automação hospitalar é uma subárea da automação que promove a automatização dos processos oriundos do ambiente hospitalar, buscando eficiência e produtividade, apropriando-se de muitos conceitos da automação industrial (Brooks e Brooks, 1998). Nesse sentido, o desenvolvimento de sistemas para o monitoramento de pacientes é uma das áreas de pesquisas da automação hospitalar que vem ganhando destaque nos últimos anos. Em relação à perspectiva dessa linha de pesquisa, os trabalhos desenvolvidos conduzem à automatização do processo de monitoramento através de sistemas que utilizam biodispositivos ou biosensores para fazer a aquisição dos sinais vitais dos pacientes (Jevon et al., 2012).

A Esclerose Lateral Amiotrófica (ELA) é uma doença neurodegenerativa, caracterizada por uma progressiva e fatal perda de neurônios motores (Yamanaka et al., 2008) do córtex cerebral, tronco encefálico e medula espinhal (Eisen, 2008), porém a atividade intelectual e cognitiva continuam íntegra. Muitos pacientes com ELA falecem por complicações da insuficiência respiratória. Devido à delicada situação do paciente com ELA, em estágios avançados da doença, faz-se necessário o uso de respiradores artificiais (Madarame et al., 2008) e um monitoramento mais eficaz e preciso para evitar sofrimento ao paciente decorrente das complicações respiratórias. Com a evolução e o agravamento da ELA, é preferível a utilização da ventilação não invasiva por pressão positiva, o BiPAP (Bilevel Positive Airway Pressure - BiPAP), pois esse método aumenta a ventilação alveolar do paciente (Poon et al., 2010), proporcionando um conforto respiratório maior e prolongando a sobrevida por muitos meses (nível A), além de poder melhorar a qualidade de vida (nível C) (Andersen et al., 2012).

O objetivo deste trabalho foi desenvolver uma arquitetura de monitoramento de pacientes e geração de alertas baseada em visão computacional para monitorar parâmetros respiratórios de pacientes com ELA no HUOL, que utilizem o equipamento de respiração artificial.

\section{Métodos}

Esse trabalho foi desenvolvido nas dependências do Hospital Universitário Onofre Lopes (HUOL) com pacientes acometidos por ELA que utilizavam um respirador artificial modelo VIVO 40 (BREAS, 2009).

\section{Arquitetura de monitoramento}

A arquitetura geral do sistema de monitoramento está dividia em 3 componentes, como mostra a Figura 1, e é constituída de um conjunto de hardware e software. Para o conjunto de hardware destacam-se para monitoramento local os seguintes componentes: Computador de placa única (Single-Board Computer-SBC), com um processador OMAP4460 de $1.2 \mathrm{Ghz}, 1 \mathrm{~Gb}$ de memória RAM (Tipo SDRAM) e conector ethernet RJ 45; Respirador artificial BiPAP modelo VIVO 40 (ventilador mecânico); Webcam Logitech modelo C210 com resolução de 1.3 MP (640x480). Para o conjunto de software destacamse os seguintes componentes: Sistema Operacional Linux Ubuntu 11.10 (modo texto para SBC); Engine Tesseract OCR (Optical Character Recognition); Biblioteca de visão computacional OpenCV (Open source computer vision).

O primeiro componente dessa arquitetura, o Leito, é formado pela SBC, que armazena o algoritmo de visão computacional para aquisição dos dados monitorados no display do BiPAP, o BiPAP e a webcam. Após o
Leito

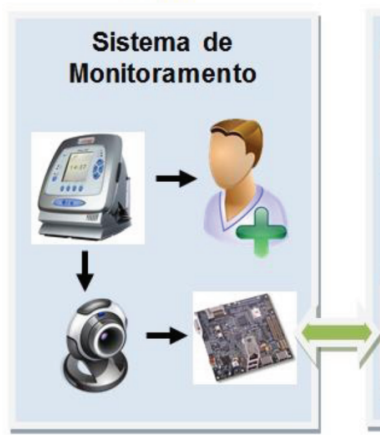

Figura 1. Arquitetura do sistema de monitoramento.
Servidor

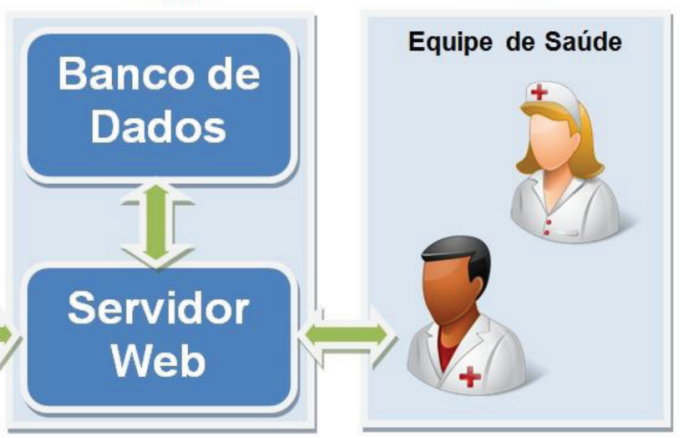

Figure 1. Architecture of the monitoring system. 
processo de aquisição, os dados são enviados para o segundo componente da arquitetura (Servidor) através de uma conexão TCP.

O segundo componente da arquitetura do sistema de monitoramento está dividido em dois subsistemas, são eles: o Sistema de Banco de Dados, que define o subsistema de armazenamento das informações de todo o processo de monitoramento. Para implementar do subsistema foi utilizado o Sistema de Gerenciamento de Banco de Dados (SGBD) PostgreSQL; e o Servidor Web, que define um Sistema Web (desenvolvido em PHP) de supervisão dos parâmetros respiratórios do paciente com ELA de forma remota.

$\mathrm{O}$ terceiro componente dessa arquitetura, o HUOL, define o cliente do sistema (e.g. fisioterapeuta, médico e enfermeira). Através de qualquer dispositivo (e.g. Smartphone, tablet ou computador) que possua um navegador, o cliente pode se conectar ao sistema de supervisão, por meio de uma conexão HTTP, para monitorar de forma on-line os parâmetros respiratórios dos pacientes com ELA.

\section{Algoritmo de extração de dados}

O algoritmo de extração de dados foi desenvolvido utilizando a biblioteca de funções de programação de visão computacional OpenCV (Open source computer vision) e a linguagem de programação $\mathrm{C}++$. O principal objetivo do algoritmo é extrair os valores dos parâmetros respiratórios dispostos no display do respirador artificial BiPAP, pois este não fornece uma interface para aquisição desses dados de forma online. Para isso foi necessário aplicar técnicas de visão computacional, tais como: filtragem de ruídos, segmentação de imagem, equalização de imagem e correlação de correspondentes.

O processo de funcionamento do algoritmo de visão computacional apresentado neste trabalho está dividido em 5 fases: pré-processamento da imagem, localização das áreas de interesse na imagem, reconhecimento de caracteres, análise de parâmetros respiratórios e envio de valores dos parâmetros para a base de dados, como mostra o diagrama de atividade na Figura 2.

$\mathrm{Na}$ fase de pré-processamento da imagem, a imagem é processada por meio de um filtro gaussiano para redução dos ruídos, posteriormente ocorrem a segmentação por limiarização e normalização dos valores de brilho para melhorar o contraste da imagem e definição das regiões de contorno.

O processo de localização (ver Figura 3) diz respeito à busca dos valores dos parâmetros respiratórios e consiste no uso de um conjunto 120 de imagens modelo (carrossel de templates) correspondentes às imagens do display do BiPAP sob diversas condições de luminosidade. A busca é feita através do "deslizamento" da imagem modelo sobre a imagem de entrada e o resultado armazenado em uma nova imagem que é enviada para a fase seguinte.

$\mathrm{Na}$ fase de reconhecimento de caracteres foi utilizada a engine Tesseract OCR (Optical Character Recognition - OCR) (TesseractOCR, 1995) para reconhecer os valores dos parâmetros nas imagens, extraí-los e armazená-los em variáveis. Ela foi escolhida devido ser multi-plataforma, possuir uma API (Application Programming Interface) bem estruturada e fácil de implementar.

$\mathrm{Na}$ fase de análise dos parâmetros é realizada uma avaliação dos valores dos parâmetros com o

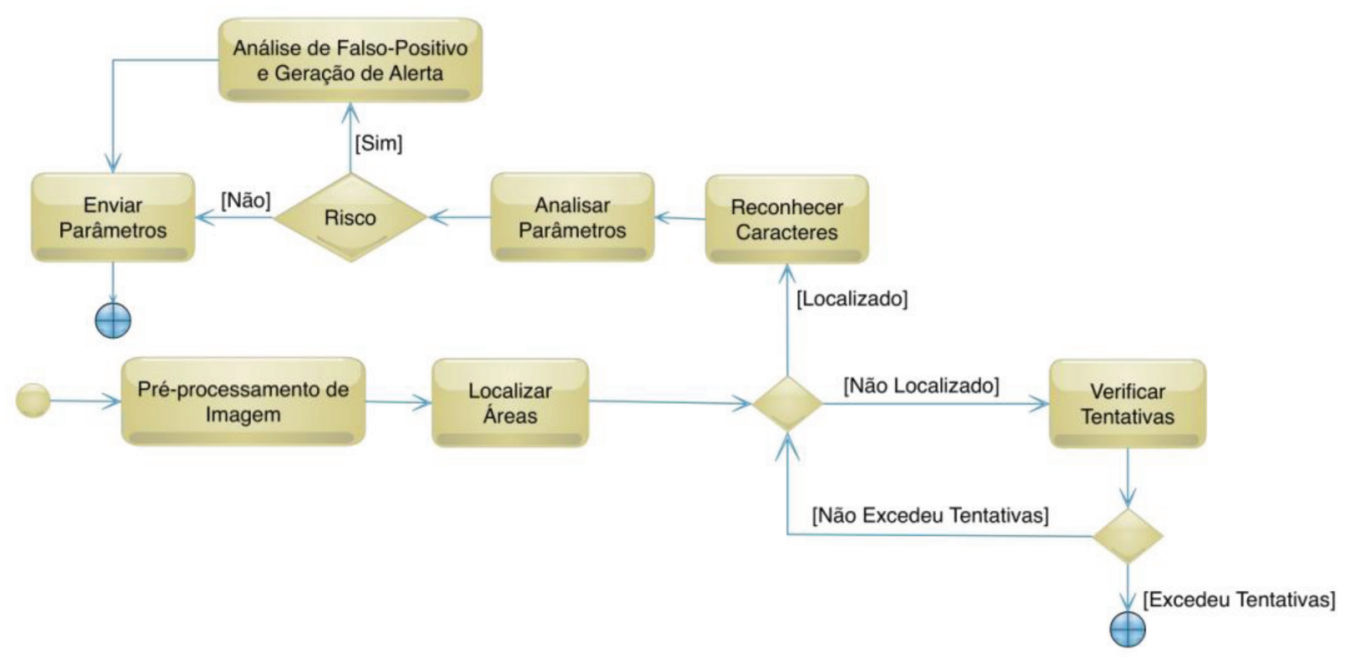

Figura 2. Diagrama de atividades do algoritmo de extração de dados.

Figure 2. Activity diagram of the extract data algorithm. 
Tabela 1. Resultados dos testes de desempenho de reconhecimento de caracteres.

Table 1. Results of the performance tests for character recognition.

\begin{tabular}{|c|c|c|c|c|c|c|c|c|c|c|c|}
\hline & \multicolumn{3}{|c|}{$\begin{array}{c}\text { Teste \#01 } \\
\text { (Interferência luminosa } \\
\text { baixa) }\end{array}$} & \multicolumn{3}{|c|}{$\begin{array}{c}\text { Teste \#02 } \\
\text { (Interferência luminosa } \\
\text { moderada) }\end{array}$} & \multicolumn{3}{|c|}{$\begin{array}{c}\text { Teste } \# 03 \\
\text { (Interferência luminosa } \\
\text { alta) }\end{array}$} & \multicolumn{2}{|c|}{ Média } \\
\hline & Amostras & $\begin{array}{c}\text { Acertos } \\
(\%)\end{array}$ & $\begin{array}{c}\text { Erros } \\
(\%)\end{array}$ & Amostras & $\begin{array}{c}\text { Acertos } \\
(\%)\end{array}$ & $\begin{array}{c}\text { Erros } \\
(\%)\end{array}$ & Amostras & $\begin{array}{c}\text { Acertos } \\
(\%)\end{array}$ & $\begin{array}{c}\text { Erros } \\
(\%)\end{array}$ & $\begin{array}{c}\text { Acertos } \\
(\%)\end{array}$ & $\begin{array}{c}\text { Erros } \\
(\%)\end{array}$ \\
\hline VC Estimado & 548 & 94,9 & 5,1 & 621 & 88,73 & 11,27 & 803 & 99,51 & 0,49 & 94,38 & 5,62 \\
\hline FR Total & 524 & 99,62 & 0,38 & 590 & 92,04 & 7,96 & 798 & 97,99 & 2,01 & 96,55 & 3,45 \\
\hline Fuga & 532 & 100,00 & 0,00 & 593 & 99,84 & 0,16 & 800 & 99,00 & 1,00 & 99,61 & 0,39 \\
\hline $\begin{array}{l}\text { Relação } \\
\text { I:E - Inspiração }\end{array}$ & 527 & 97,44 & 2,56 & 588 & 91,37 & 8,63 & 791 & 86,86 & 13,14 & 91,89 & 8,11 \\
\hline $\begin{array}{l}\text { Relação } \\
\text { I:E - Expiração }\end{array}$ & 527 & 96,85 & 3,15 & 588 & 92,36 & 7,64 & 791 & 86,98 & 13,02 & 92,06 & 7,94 \\
\hline $\begin{array}{l}\text { Média de A/E } \\
(\%)\end{array}$ & - & 97,76 & 2,24 & - & 92,87 & 7,13 & - & 94,07 & 5,93 & 94,90 & 5,10 \\
\hline $\begin{array}{l}\text { Total de } \\
\text { amostras }\end{array}$ & 2658 & - & - & 2980 & - & - & 3983 & - & - & - & - \\
\hline
\end{tabular}

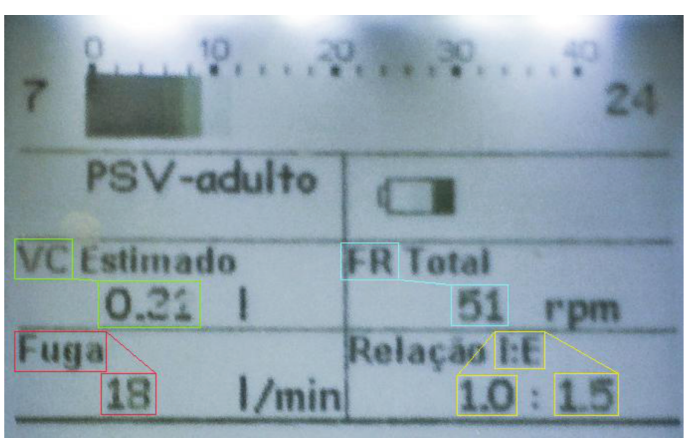

Figura 3. Deslocamento de pontos para encontrar a área do valor do parâmetro.

Figure 3. Shifting points to locate the area containing the value of a parameter.

objetivo de verificar situações de risco ao paciente e geração de alertas (dispositivos móveis e supervisório web). Como a variação desses valores não acontece de forma abrupta, o disparo dos alertas passa por um mecanismo de filtragem que evidencia a real ocorrência do alerta ou um falso-positivo. Além disso, é importante ressaltar que sempre é enviada uma imagem do display do BiPAP para apreciação da equipe de saúde.

\section{Cenários de teste}

Para a realização dos testes foram criados três cenários sob diferentes condições de luminosidade. O primeiro teste foi realizado permitindo apenas a entrada da luz proveniente da janela do leito (salientando que o teste foi realizado durante o dia) mantendo-se todas as luzes do leito apagadas. No segundo teste, somente metade das luzes se mantiveram acesas, além da entrada de luz do meio externo. No terceiro e último teste, todas as luzes do leito se mantiveram acesas, além da entrada de luz do meio externo.

\section{Resultados}

O sistema de monitoramento passou por avaliações de desempenho, com foco na precisão e a eficiência de acertos do sistema de visão computacional. A Tabela 1 apresenta a comparação entre os resultados obtidos durante a execução da etapa de reconhecimento de caracteres.

\section{Discussão}

Durante a execução dos testes, a etapa de reconhecimento de caracteres (Tabela 1) obteve uma média geral de acertos de $94.90 \%$. Além disso, quando a interferência luminosa foi mínima, o sistema obteve uma média geral de acertos de 97,76\%. Porém, observamos que para os parâmetros Relação I:E - Inspiração : Expiração - apresentou uma taxa geral de erros de $8,11 \%$ e $7,94 \%$. Para o terceiro teste, apesar de ter obtido um média geral de acertos maior que o segundo, é possível notar que a incidência de ruídos de luz comprometeu o desempenho de acertos dos parâmetros anteriormente mencionados tendo uma taxa de erros superior a 10\%. É importante destacar que a opção por utilizar visão computacional para o processamento de imagens ocorreu em função dos ventiladores mecânicos disponíveis para pesquisa, bem como, os encontrados no mercado, não permitirem uma leitura on-line dos dados e também não adotarem um a padrão aberto para leitura dos dados produzidos. Deste modo, a utilização de visão computacional foi considerada uma solução bastante razoável, pois os impactos financeiros são bem menores, do que se 
fosse desenvolvido um ventilador mecânico com esse propósito. Outro fator é que os equipamentos utilizados já estão validados e certificados pelos órgãos competentes.

Desta forma o sistema de monitoramento de pacientes com ELA no ambiente hospitalar possibilitou uma ação mais eficaz da equipe de saúde em situações risco para os pacientes. Os profissionais da saúde que estejam atuando junto ao paciente podem monitorar os parâmetros respiratórios do mesmo remotamente, por meio de dispositivos com acesso a internet, tais como: SmartPhone, Tablet, SmartTV, Notebook entre outros. Nos casos de situações de risco do paciente, a equipe de saúde recebeu alertas através de mensagens enviadas para os celulares, bem como na tela do sistema de supervisão web, o qual emite um alarme sonoro para avisar sobre o risco. No que se refere ao desempenho do sistema no tocante a taxa de acertos do mesmo, é possível notar que o mesmo tem o comportamento melhorado quando a interferência luminosa externa é reduzida. Dessa forma, o desenvolvimento de um suporte para fixação da webcam envolvendo-a e eliminando a interferência externa tende a gerar resultados melhores que os apresentados nesse trabalho. Além disso, a expansão desse sistema para atendimento homecare é uma evolução natural do mesmo.

\section{Agradecimentos}

Ao Laboratório de Inovação Tecnológica em Saúde (LAIS/UFRN), assim como, à CAPES e ao CNPq pelo fomento a este projeto de pesquisa.

\section{Referências}

Andersen PM, Abrahams S, Borasio GD, Carvalho M, Chio A, Damme PV, Hardiman O, Kollewe K, Morrison KE, Petri S, Pradat PF, Silani V, Tomik B, Wasner M, Weber M. EFNS guidelines on the Clinical Management of Amyotrophic Lateral Sclerosis (MALS) - revised report of an EFNS task force. European Journal of Neurology. 2012; 19(3):360-75. PMid:21914052. http://dx.doi.org/10.1111/j.14681331.2011.03501.x

Breas. Vivo 40: Manual do utilizador. Lisboa; 2009.

Brooks J, Brooks L. Automation in the medical field. Engineering in Medicine and Biology Magazine. 1998; 17(4).

Eisen A. Amyotrophic lateral sclerosis: A 40year personal perspective. Journal of Clinical Neuroscience. 2009; 16(4):505-12. PMid:19231200. http:// dx.doi.org/10.1016/j.jocn.2008.07.072

Jevon P, Ewens B, Pooni JS. Monitoring the critically ill patient. 3th ed. Londres: Wiley-Blackwell; 2012.

Madarame T, Inoue T, Shino M, Tanaka H, Kamata M. The development of a brain computer interface device for amyotrophic lateral sclerosis patients. In: IEEE International Conference on Systems, Man and Cybernetics 2008: Proceedings of the International Conference on Systems, Man and Cybernetics; 2008 out 12-15; Singapore. Nova York: IEEE; 2008. p. 2401-6.

Poon CA, Becker KA, Littner MR. Noninvasive positive airwaypressure in Hypercapnic respiratory failure in noncardiac medical disorders. Sleep Medicine Clinics. 2010; 5(3):451-70. http://dx.doi.org/10.1016/j. jsmc.2010.05.008

Tesseract OCR. Engine Tesseract OCR (Optical Character Recognition). 1995. [cited 2011 Nov 06]. Available from: http://code.google.com/p/tesseract-ocr/.

Valentim RAM, Araújo BG, Lacerda JMT, Souza PCO, Carvalho TPM. Automação hospitalar: O estado da arte. Revista Brasileira de Inovação Tecnológica em Saúde. 2012; 1(1):27-38.

Yamanaka K, Chun SJ, Boillee S, Fujimori-Tonou N, Yamashita H, Gutmann DH, Takahashi R, Misawa H, Cleveland DW. Astrocytes as determinants of disease progression in inherited amyotrophic lateral sclerosis. Nature Neuroscience Journal. 2008; 11(3):251-3. PMid:18246065 PMCid:3137510. http://dx.doi.org/10.1038/nn2047

\footnotetext{
Autores

Gustavo Henrique Souto da Silva, Antônio Higor Freire de Morais*, Ricardo Alexsandro de Medeiros Valentim, Rummenigge Rudson Dantas, Leila Raulino Câmara Cavalcanti, Mário Emílio Teixeira Dourado Júnior Laboratório de Inovação Tecnológica em Saúde, Hospital Universitário Onofre Lopes - HUOL. Laboratório Natalnet, Programa de Pós-graduação em Engenharia Elétrica e Computação - PPGEEC, Universidade Federal do Rio Grande do Norte - UFRN, Av. Nilo Peçanha, 620, Petrópolis, CEP 59012-300, Natal, RN, Brasil.
} 